\title{
All-optical machine learning using diffractive deep neural networks
}

\author{
Xing Lin ${ }^{1,2,3 *}$, Yair Rivenson ${ }^{1,2,3 *}$, Nezih T. Yardimci ${ }^{1,3}$, Muhammed Veli $^{1,2,3}$, Yi Luo $^{1,2,3}$, Mona Jarrahi $^{1,3}$, Aydogan Ozcan $^{1,2,3,4} \dagger$ \\ ${ }^{1}$ Electrical and Computer Engineering Department, University of California, Los Angeles, CA, 90095, USA. 'Bioengineering Department, University of California, Los Angeles, \\ CA, 90095, USA. ${ }^{3}$ California NanoSystems Institute (CNSI), University of California, Los Angeles, CA, 90095, USA. ${ }^{4}$ Department of Surgery, David Geffen School of \\ Medicine, University of California, Los Angeles, CA, 90095, USA.
}

*These authors contributed equally to this work.

†Corresponding author. Email: ozcan@ucla.edu

Deep learning has been transforming our ability to execute advanced inference tasks using computers. We introduce a physical mechanism to perform machine learning by demonstrating an all-optical Diffractive Deep Neural Network ( $D^{2} N N$ ) architecture that can implement various functions following the deep learning-based design of passive diffractive layers that work collectively. We create 3D-printed $D^{2} N N s$ that implement classification of images of handwritten digits and fashion products as well as the function of an imaging lens at terahertz spectrum. Our all-optical deep learning framework can perform, at the speed of light, various complex functions that computer-based neural networks can implement, and will find applications in all-optical image analysis, feature detection and object classification, also enabling new camera designs and optical components that perform unique tasks using $D^{2} \mathrm{NNs}$.

Deep learning is one of the fastest-growing machine learning methods (1), and it uses multi-layered artificial neural networks implemented in a computer to digitally learn data representation and abstraction, and perform advanced tasks, comparable to or even superior than the performance of human experts. Recent examples where deep learning has made major advances in machine learning include medical image analysis (2), speech recognition (3), language translation (4), image classification (5), among others $(1,6)$. Beyond some of these mainstream applications, deep learning methods are also being used for solving inverse imaging problems (7-13).

We introduce an all-optical deep learning framework, where the neural network is physically formed by multiple layers of diffractive surfaces that work in collaboration to optically perform an arbitrary function that the network can statistically learn. While the inference/prediction of the physical network is all-optical, the learning part that leads to its design is done through a computer. We term this framework as Diffractive Deep Neural Network $\left(D^{2} \mathrm{NN}\right)$ and demonstrate its inference capabilities through both simulations and experiments. Our $\mathrm{D}^{2} \mathrm{NN}$ can be physically created by using several transmissive and/or reflective layers (14), where each point on a given layer either transmits or reflects the incoming wave, representing an artificial neuron that is connected to other neurons of the following layers through optical diffraction (Fig. 1A). Following Huygens' Principle, our terminology is based on each point on a given layer acting as a secondary source of a wave, the amplitude and phase of which are determined by the product of the input wave and the complex- valued transmission or reflection coefficient at that point; see (14) for an analysis of the waves within a $\mathrm{D}^{2} \mathrm{NN}$. Therefore, an artificial neuron in a $\mathrm{D}^{2} \mathrm{NN}$ is connected to other neurons of the following layer through a secondary wave that is modulated in amplitude and phase by both the input interference pattern created by the earlier layers and the local transmission/reflection coefficient at that point. As an analogy to standard deep neural networks (Fig. 1D), one can consider the transmission/reflection coefficient of each point/neuron as a multiplicative "bias" term, which is a learnable network parameter that is iteratively adjusted during the training process of the diffractive network, using an error back-propagation method. After this numerical training phase, the $\mathrm{D}^{2} \mathrm{NN}$ design is fixed and the transmission/reflection coefficients of the neurons of all the layers are determined. This $\mathrm{D}^{2} \mathrm{NN}$ design, once physically fabricated using e.g., 3D-printing, lithography, etc., can then perform, at the speed of light, the specific task that it is trained for, using only optical diffraction and passive optical components/layers that do not need power, creating an efficient and fast way of implementing machine learning tasks.

In general, phase and amplitude of each neuron can be a learnable parameter, providing a complex-valued modulation at each layer, which improves the inference performance of the diffractive network (fig. S1) (14). For coherent transmissive networks with phase-only modulation, each layer can be approximated as a thin optical element (Fig. 1). Through deep learning, the phase values of the neurons of each layer of the diffractive network are iteratively adjusted (trained) to 
perform a specific function by feeding training data at the input layer and then computing the network's output through optical diffraction. Based on the calculated error with respect to the target output, determined by the desired function, the network structure and its neuron phase values are optimized using an error back-propagation algorithm, which is based on the stochastic gradient descent approach used in conventional deep learning (14).

To demonstrate the performance of $\mathrm{D}^{2} \mathrm{NN}$ framework, we first trained it as a digit classifier to perform automated classification of handwritten digits, from zero to nine (Figs. 1B and $2 \mathrm{~A}$ ). For this task, phase-only transmission masks were designed by training a 5-layer $\mathrm{D}^{2} \mathrm{NN}$ with 55,000 images (5,000 validation images) from MNIST (Modified National Institute of Standards and Technology) handwritten digit database (15). Input digits were encoded into the amplitude of the input field to the $\mathrm{D}^{2} \mathrm{NN}$, and the diffractive network was trained to map input digits into ten detector regions, one for each digit. The classification criterion was to find the detector that has the maximum optical signal and this was also used as a loss function during the network training (14).

After its training, the design of the $\mathrm{D}^{2} \mathrm{NN}$ digit classifier was numerically tested using 10,000 images from MNIST test dataset (which were not used as part of the training or validation image sets) and achieved a classification accuracy of 91.75\% (Fig. 3C and fig. S1). In addition to the classification performance of the diffractive network, Fig. $3 \mathrm{C}$ also reports the energy distribution observed at the network output plane for the same 10,000 test digits, the results of which clearly demonstrate that the diffractive network learned to focus the input energy of each handwritten digit into the correct (i.e., the target) detector region that it was trained for. As reported in figs. S1 and S2, by using complex-valued modulation and increasing the number of layers, neurons and connections in the diffractive network, our classification accuracy can be further improved; for example, figure S2 demonstrates a Legolike physical transfer learning behavior for $\mathrm{D}^{2} \mathrm{NN}$ framework, where the inference performance of an already existing $\mathrm{D}^{2} \mathrm{NN}$ can be further improved by adding new diffractive layers to it or in some cases by peeling off (i.e., discarding) some of the existing layers, where the new layers to be added are trained for improved inference (coming from the entire diffractive network: old and new layers). Using a patch of 2 layers added to an existing and fixed $\mathrm{D}^{2} \mathrm{NN}$ design $(\mathrm{N}=5)$, we improved our MNIST classification accuracy to $93.39 \%$ (fig. S2) (14); the state-of-the-art convolutional neural network performance has been reported as $99.60 \%-99.77 \%$ (16-18). More discussion on reconfiguring $\mathrm{D}^{2} \mathrm{NN}$ designs is provided in (14).

Following these numerical results, we 3D-printed our 5layer $\mathrm{D}^{2} \mathrm{NN}$ design (Fig. 2A), with each layer having an area of $8 \times 8 \mathrm{~cm}$, followed by ten detector regions defined at the output plane of the diffractive network (Figs. 1B and 3A), and tested its inference performance using continuous wave illumination at $0.4 \mathrm{THz}$ (Figs. 2, C and D). Phase values of each layer's neurons were physically encoded using the relative thickness of each 3D-printed neuron. Numerical testing of this 5-layer $\mathrm{D}^{2} \mathrm{NN}$ design achieved a classification accuracy of $91.75 \%$ over $\sim 10,000$ test images (Fig. $3 \mathrm{C}$ ), and to quantify the match between these numerical testing results and our experiments, we 3D-printed 50 handwritten digits i.e., 5 different inputs per digit, selected among the same $91.75 \%$ of the test images that numerical testing was successful. For each input object that is uniformly illuminated with the $\mathrm{THz}$ source, we imaged the output plane of the $\mathrm{D}^{2} \mathrm{NN}$ to map the intensity distribution for each detector region that is assigned to a digit. The results (Fig. 3B) demonstrate the success of the 3D-printed diffractive neural network and its inference capability: the average intensity distribution at the output plane of the network for each input digit clearly reveals that the $3 \mathrm{D}$-printed $\mathrm{D}^{2} \mathrm{NN}$ was able to focus the input energy of the beam and achieve a maximum signal at the corresponding detector region that was assigned for that digit. Despite 3D-printing errors, possible alignment issues and other experimental error sources in our set-up (see (14)), the match between the experimental and numerical testing of our 5 -layer $\mathrm{D}^{2} \mathrm{NN}$ design was found to be $88 \%$ (Fig. 3B). This relatively small reduction in the performance of the experimental network compared to our numerical testing is especially more pronounced for the digit " 0 " since it is challenging to 3D-print the large void region at the center of the digit; similar printing challenges were also observed for other digits that have void regions, e.g., "6", "8", "9" (Fig. 3B).

Next, we tested the classification performance of $\mathrm{D}^{2} \mathrm{NN}$ framework with a more complicated image dataset, i.e., the Fashion MNIST (19), which includes ten classes, each representing a fashion product (t-shirts, trousers, pullovers, dresses, coats, sandals, shirts, sneakers, bags, and ankle boots; see fig. S3 for sample images). In general, for a coherently illuminated $\mathrm{D}^{2} \mathrm{NN}$ we can use the amplitude and/or phase channels of the input plane to represent data to be classified. In our digit classification results reported earlier, input objects were encoded using the amplitude channel, and to demonstrate the utility of the phase channel of the network input, we encoded each input image corresponding to a fashion product as a phase-only object modulation (14). Our $\mathrm{D}^{2} \mathrm{NN}$ inference results (as a function of the number of layers, neurons and connections) for classification of fashion products are summarized in figs. S4 and S5. To provide an example of its performance, a phase-only and a complex-valued modulation $\mathrm{D}^{2} \mathrm{NN}$ with $\mathrm{N}=5$ diffractive layers (sharing the same physical network dimensions as the digit classification $\mathrm{D}^{2} \mathrm{NN}$ shown in Fig. 2A) reached an accuracy of $81.13 \%$ and $86.33 \%$, respectively (fig. S4). By increasing the number of diffractive layers to $\mathrm{N}=10$ and the total number of neurons 
to 0.4 million, our classification accuracy increased to $86.60 \%$ (fig. S5). For convolutional neural net based standard deep learning, the state-of-the-art performance for Fashion MNIST classification accuracy has been reported as $96.7 \%$, using $~ 8.9$ million learnable parameters and $\sim 2.5$ million neurons $(20)$.

To experimentally demonstrate the performance of fashion product classification using a physical $\mathrm{D}^{2} \mathrm{NN}$, we $3 \mathrm{D}-$ printed our phase-only 5 -layer design and 50 fashion products used as test objects (i.e., 5 per class) based on the same procedures employed for digit classification diffractive network (Figs. 2A and 3), except that each input object information was encoded in the phase channel. Our results are summarized in Fig. 4, revealing a 90\% match between the experimental and numerical testing of our 5 -layer $\mathrm{D}^{2} \mathrm{NN}$ design, with 5 errors out of 50 fashion products. Compared to digit classification ( 6 errors out of 50 digits, Fig. 3), here we got a slightly better match between the experimental and numerical testing results (despite the more challenging nature of Fashion MNIST dataset), which might be related to the fact that we used the phase channel to encode input image information for fashion products, which does not suffer from the challenges associated with 3D-printing of void regions, such as in digits "0", "6", " 8 ", and "9" (Fig. 3).

Next, we tested the performance of a phase-only $\mathrm{D}^{2} \mathrm{NN}$, composed of five 3D-printed transmission layers (Fig. 2B), which was trained using ImageNet database (21) to create a unit-magnification image of the input optical field amplitude at its output plane $(\sim 9 \times 9 \mathrm{~cm})$, i.e., the output image has the same physical size as the input object (14). As illustrated in fig. S6, A and C, the trained network initially connects every single amplitude point at the input plane to various neurons and features of the following layers, which then focus the light back to a point at the output (i.e., image) plane, which is, as expected, quite different than the case of free-space diffraction (i.e., without the presence of the diffractive network), illustrated in fig. S6, B and D.

After its training and blind testing, numerically proving the imaging capability of the network as shown in figs. S6 and S7, next we 3D-printed this designed $\mathrm{D}^{2} \mathrm{NN}$. Using the same experimental set-up shown in Fig. 2, C and D, we imaged the output plane of the $3 \mathrm{D}$-printed $\mathrm{D}^{2} \mathrm{NN}$ for various input objects that were uniformly illuminated by continuous wave radiation at $0.4 \mathrm{THz}$. Fig. S8 summarizes our experimental results achieved with this 3D-printed $\mathrm{D}^{2} \mathrm{NN}$, which successfully projected unit-magnification images of the input patterns at the output plane of the network, learning the function of an imaging lens, or a physical auto-encoder. To evaluate the point spread function of this $\mathrm{D}^{2} \mathrm{NN}$, we imaged pinholes with different diameters ( $1 \mathrm{~mm}, 2 \mathrm{~mm}$ and $3 \mathrm{~mm}$ ), which resulted in output images, each with a full-width-athalf-maximum (FWHM) of $1.5 \mathrm{~mm}, 1.4 \mathrm{~mm}$ and $2.5 \mathrm{~mm}$, respectively (fig. S8B). Our results also revealed that the printed network can resolve a line-width of $1.8 \mathrm{~mm}$ at $0.4 \mathrm{THz}$ (corresponding to a wavelength of $0.75 \mathrm{~mm}$ in air), which is slightly worse in resolution compared to the numerical testing of our $\mathrm{D}^{2} \mathrm{NN}$ design, where the network could resolve a line-width of $\sim 1.2 \mathrm{~mm}$ (fig. S7C). This experimental degradation in the performance of the diffractive network can be due to e.g., 3D-printing errors, potential misalignments and absorption related losses in the 3D-printed network (also see (14)).

Optical implementation of learning in artificial neural networks is promising due to the parallel computing capability and power efficiency of optical systems (22-24). Compared to previous opto-electronics based learning approaches $(22,25-27)$, the $\mathrm{D}^{2} \mathrm{NN}$ framework provides a unique all-optical deep learning engine that efficiently operates at the speed of light using passive components and optical diffraction. An important advantage of $\mathrm{D}^{2} \mathrm{NNs}$ is that they can be easily scaled up using various high-throughput and large-area 3D fabrication methods (e.g., soft-lithography, additive manufacturing) and wide-field optical components and detection systems, to cost-effectively reach tens to hundreds of millions of neurons and hundreds of billions of connections in a scalable and power-efficient manner. For example, integration of $\mathrm{D}^{2} \mathrm{NNs}$ with lensfree on-chip imaging systems $(28,29)$ could provide extreme parallelism within a cost-effective and portable platform. Such large-scale $\mathrm{D}^{2} \mathrm{NNs}$ might be transformative for various applications, including image analysis, feature detection, object classification, and might also enable new microscope or camera designs that can perform unique imaging tasks using $\mathrm{D}^{2} \mathrm{NNs}$. To achieve these, a monolithic $\mathrm{D}^{2} \mathrm{NN}$ design that combines all the layers of the network as part of a 3D fabrication method would be desirable. Among other techniques, laser lithography based on two-photon polymerization (30) can provide a desired solution for creating such monolithic $\mathrm{D}^{2} \mathrm{NNs}$.

\section{REFERENCES AND NOTES}

1. Y. LeCun, Y. Bengio, G. Hinton, Deep learning. Nature 521, 436-444 (2015). doi:10.1038/nature14539 Medline

2. G. Litjens, T. Kooi, B. E. Bejnordi, A. A. A. Setio, F. Ciompi, M. Ghafoorian, J. A. W. M. van der Laak, B. van Ginneken, C. I. Sánchez, A survey on deep learning in medical image analysis. Med. Image Anal. 42, 60-88 (2017). doi:10.1016/j.media.2017.07.005 Medline

3. A. Graves, A. Mohamed, G. Hinton, in IEEE Conference on Acoustics, Speech and Signal Processing (2013), pp. 6645-6649.

4. K. Cho et al., Learning phrase representations using RNN encoder-decoder for statistical machine translation. arXiv preprint arXiv:1406.1078 (2014).

5. A. Krizhevsky, I. Sutskever, G. E. Hinton, in Advances in Neural Information Processing Systems (2012), pp. 1097-1105.

6. D. Silver, A. Huang, C. J. Maddison, A. Guez, L. Sifre, G. van den Driessche, J. Schrittwieser, I. Antonoglou, V. Panneershelvam, M. Lanctot, S. Dieleman, D. Grewe, J. Nham, N. Kalchbrenner, I. Sutskever, T. Lillicrap, M. Leach, K. Kavukcuoglu, T. Graepel, D. Hassabis, Mastering the game of Go with deep neural networks and tree search. Nature 529, 484-489 (2016). doi:10.1038/nature16961 Medline 
7. U. S. Kamilov, I. N. Papadopoulos, M. H. Shoreh, A. Goy, C. Vonesch, M. Unser, D. Psaltis, Learning approach to optical tomography. Optica 2, 517 (2015). doi:10.1364/OPTICA.2.000517

8. Y. Rivenson, Z. Göröcs, H. Günaydin, Y. Zhang, H. Wang, A. Ozcan, Deep learning microscopy. Optica 4, 1437 (2017). doi:10.1364/OPTICA.4.001437

9. K. H. Jin, M. T. McCann, E. Froustey, M. Unser, Deep convolutional neural network for inverse problems in imaging. IEEE Trans. Image Process. 26, 4509-4522 (2017). doi:10.1109/TIP.2017.2713099 Medline

10. Y. Rivenson, Y. Zhang, H. Gunaydin, D. Teng, A. Ozcan, Phase recovery and holographic image reconstruction using deep learning in neural networks. Light Sci. Appl. 7, 17141 (2018). doi:10.1038//sa.2017.141

11. A. Sinha, J. Lee, S. Li, G. Barbastathis, Lensless computational imaging through deep learning. Optica 4, 1117 (2017). doi:10.1364/OPTICA.4.001117

12. K. Hammernik, T. Klatzer, E. Kobler, M. P. Recht, D. K. Sodickson, T. Pock, F. Knoll, Learning a variational network for reconstruction of accelerated MRI data. Magn. Reson. Med. 79, 3055-3071 (2018). doi:10.1002/mrm.26977 Medline

13. Y. Rivenson, H. Ceylan Koydemir, H. Wang, Z. Wei, Z. Ren, H. Günaydın, Y. Zhang, Z. Göröcs, K. Liang, D. Tseng, A. Ozcan, Deep learning enhanced mobile-phone microscopy. ACS Photonics 5, 2354-2364 (2018). doi:10.1021/acsphotonics.8b00146

14. Materials and methods are available as supplementary materials.

15. Y. Lecun, L. Bottou, Y. Bengio, P. Haffner, Gradient-based learning applied to document recognition. Proc. IEEE 86, 2278-2324 (1998). doi:10.1109/5.726791

16. D. Ciregan, U. Meier, J. Schmidhuber, in IEEE Conference on Computer Vision and Pattern Recognition (2012), pp. 3642-3649.

17. C.-Y. Lee, P. W. Gallagher, Z. Tu, in Artificial Intelligence and Statistics (2016), pp. 464-472.

18. M. A. Ranzato, C. Poultney, S. Chopra, Y. LeCun, in Advances in Neural Information Processing Systems (2007), pp. 1137-1144

19. github.com/zalandoresearch/fashion-mnist.

20. github.com/ajbrock.

21. www.image-net.org

22. Y. Shen, N. C. Harris, S. Skirlo, M. Prabhu, T. Baehr-Jones, M. Hochberg, X. Sun, S. Zhao, H. Larochelle, D. Englund, M. Soljačić, Deep learning with coherent nanophotonic circuits. Nat. Photonics 11, 441-446 (2017). doi:10.1038/nphoton.2017.93

23. D. Psaltis, D. Brady, X.-G. Gu, S. Lin, Holography in artificial neural networks. Nature 343, 325-330 (1990). doi:10.1038/343325a0 Medline

24. K. H. Wagner, in OSA Frontiers in Optics (2017), pp. FW2C-1.

25. B. J. Shastri et al., Principles of neuromorphic photonics. arXiv preprint arXiv:1801.00016 (2017)

26. M. Hermans, M. Burm, T. Van Vaerenbergh, J. Dambre, P. Bienstman, Trainable hardware for dynamical computing using error backpropagation through physical media. Nat. Commun. 6, 6729 (2015). doi:10.1038/ncomms 7729 Medline

27. D. Brunner, M. C. Soriano, C. R. Mirasso, I. Fischer, Parallel photonic information processing at gigabyte per second data rates using transient states. Nat. Commun. 4, 1364 (2013). doi:10.1038/ncomms2368 Medline

28. A. Greenbaum, W. Luo, T.-W. Su, Z. Göröcs, L. Xue, S. O. Isikman, A. F. Coskun, 0. Mudanyali, A. Ozcan, Imaging without lenses: Achievements and remaining challenges of wide-field on-chip microscopy. Nat. Methods 9, 889-895 (2012). doi:10.1038/nmeth.2114 Medline

29. A. Ozcan, E. McLeod, Lensless imaging and sensing. Annu. Rev. Biomed. Eng. 18, 77-102 (2016). doi:10.1146/annurev-bioeng-092515-010849 Medline

30. M. Emons, K. Obata, T. Binhammer, A. Ovsianikov, B. N. Chichkov, U. Morgner, Two-photon polymerization technique with sub-50 nm resolution by sub-10 fs laser pulses. Opt. Mater. Express 2, 942-947 (2012). doi:10.1364/OME.2.000942

31. D. P. Kingma, J. Ba, Adam: A method for stochastic optimization. arXiv preprint arXiv:1412.6980 (2014).

32. M. Kazhdan, H. Hoppe, Screened poisson surface reconstruction. ACM Trans. Graph. 32, 1-13 (2013). doi:10.1145/2487228.2487237

33. http://www.meshlab.net

34. P. Cignoni et al., Meshlab: an open-source mesh processing tool. in Eurographics Italian Chapter Conference (2008), pp. 129-136.

35. J. W. Goodman, Introduction to Fourier optics (Roberts and Company Publishers, 2005)
36. C. Trabelsi et al., Deep complex networks. arXiv preprint arXiv:1705.09792 (2017).

37. Z. Wang, A. C. Bovik, H. R. Sheikh, E. P. Simoncelli, Image quality assessment: From error visibility to structural similarity. IEEE Trans. Image Process. 13, 600612 (2004). doi:10.1109/TIP.2003.819861 Medline

38. N. Srivastava, G. Hinton, A. Krizhevsky, I. Sutskever, R. Salakhutdinov, Dropout: A simple way to prevent neural networks from overfitting. J. Mach. Learn. Res. 15, 1929-1958 (2014).

39. V. Bianchi, T. Carey, L. Viti, L. Li, E. H. Linfield, A. G. Davies, A. Tredicucci, D. Yoon, P. G. Karagiannidis, L. Lombardi, F. Tomarchio, A. C. Ferrari, F. Torrisi, M. S. Vitiello, Terahertz saturable absorbers from liquid phase exfoliation of graphite. Nat. Commun. 8, 15763 (2017). doi:10.1038/ncomms15763 Medline

40. A. Marini, J. D. Cox, F. J. García de Abajo, Theory of graphene saturable absorption. Phys. Rev. B 95, 125408 (2017). doi:10.1103/PhysRevB.95.125408

41. X. Yin, T. Feng, Z. Liang, J. Li, Artificial Kerr-type medium using metamaterials. Opt. Express 20, 8543-8550 (2012). doi:10.1364/0E.20.008543 Medline

42. Y. Xiao, H. Qian, Z. Liu, Nonlinear metasurface based on giant optical kerr response of gold quantum wells. ACS Photonics 5, 1654-1659 (2018). doi:10.1021/acsphotonics.7b01140

43. N. Yu, F. Capasso, Flat optics with designer metasurfaces. Nat. Mater. 13, 139-150 (2014). doi:10.1038/nmat3839 Medline

44. M. Khorasaninejad, W. T. Chen, R. C. Devlin, J. Oh, A. Y. Zhu, F. Capasso, Metalenses at visible wavelengths: Diffraction-limited focusing and subwavelength resolution imaging. Science 352, 1190-1194 (2016) doi:10.1126/science.aaf6644 Medline

45. A. V. Kildishev, A. Boltasseva, V. M. Shalaev, Planar photonics with metasurfaces, Science 339, 1232009 (2013). doi:10.1126/science.1232009 Medline

46. D. Grischkowsky, S. Keiding, M. Van Exter, C. Fattinger, Far-infrared time-domain spectroscopy with terahertz beams of dielectrics and semiconductors. J. Opt. Soc. Am. B 7, 2006-2015 (1990). doi:10.1364/JOSAB.7.002006

\section{ACKNOWLEDGMENTS}

The authors acknowledge D. Mengu, Z. Wei, X. Wang and Y. Xiao of UCLA for their assistance with coding. Funding: The Ozcan Group at UCLA acknowledges the support of the National Science Foundation and the Howard Hughes Medical Institute. Author contributions: A.O., X.L. and Y.R. conceived the research, X.L., N.Y., Y.L., Y.R., M.V. and M.J. contributed to the experiments, X.L., N.Y., M.V. and Y.R. processed the data. A.O., X.L., M.V., N.Y., Y.R., Y.L., and M.J. prepared the manuscript. A.O. initiated and supervised the research. Competing interests: A.O., X.L. and Y.R. are inventors of a patent application on D²NN. Data and materials availability: All the data and methods are present in the main text and the supplementary materials.

\section{SUPPLEMENTARY MATERIALS}

www.sciencemag.org/cgi/content/full/science.aat8084/DC1

Materials and Methods

Figs. S1 to S16

References (31-46)

6 April 2018; accepted 12 July 2018

Published online 26 July 2018

10.1126/science.aat8084 

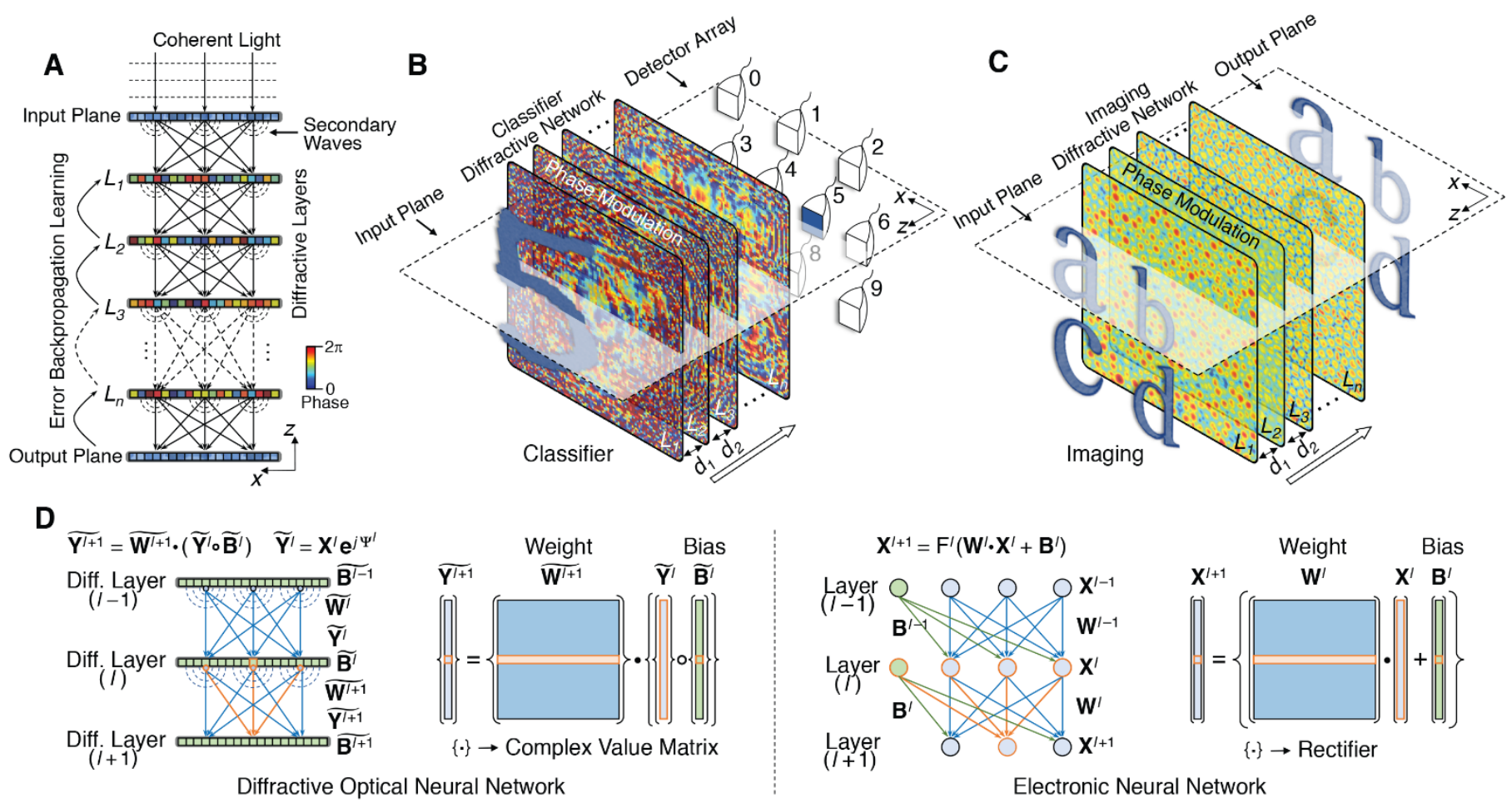

Electronic Neural Network

Fig. 1. Diffractive Deep Neural Networks (D²NN). (A) D²NN comprises multiple transmissive (or reflective) layers, where each point on a given layer acts as a neuron, with a complex-valued transmission (or reflection) coefficient. These transmission/reflection coefficients of each layer can be trained using deep learning to perform a function between the input and output planes of the network. After this learning phase, the $D^{2} N N$ design is fixed, and once it is fabricated or 3D-printed, it performs the learned function at the speed of light. We trained and experimentally implemented different types of $D^{2} N N s$ : (B) classifier (for handwritten digits and fashion products) and (C) imaging lens. (D) A comparison between $D^{2} \mathrm{NN}$ and a conventional neural network is presented (also see (14)). Based on coherent waves, $D^{2} N N$ operates on complex-valued inputs, with multiplicative bias terms. Weights in a $D^{2} N N$ are based on free-space diffraction and determine the coherent interference of the secondary waves that are phase and/or amplitude modulated by the previous layers. "o" refers to a Hadamard product operation. Electronic neural network refers to the conventional neural network virtually implemented in computer. 

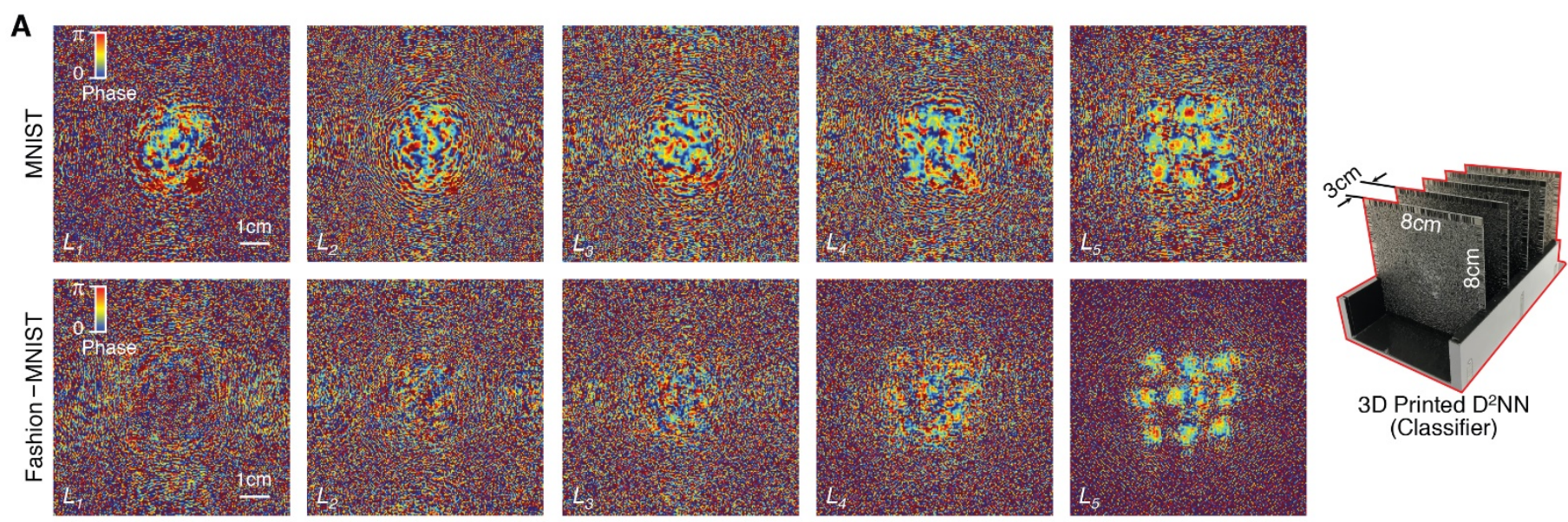

3D Printed D'NN (Classifier)

Trained Multi-Layer Phase Mask (Classifier)
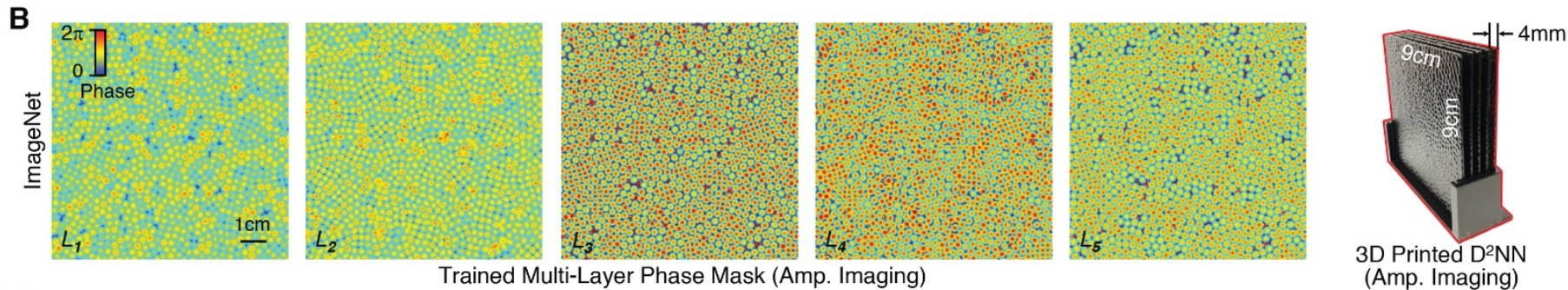

C

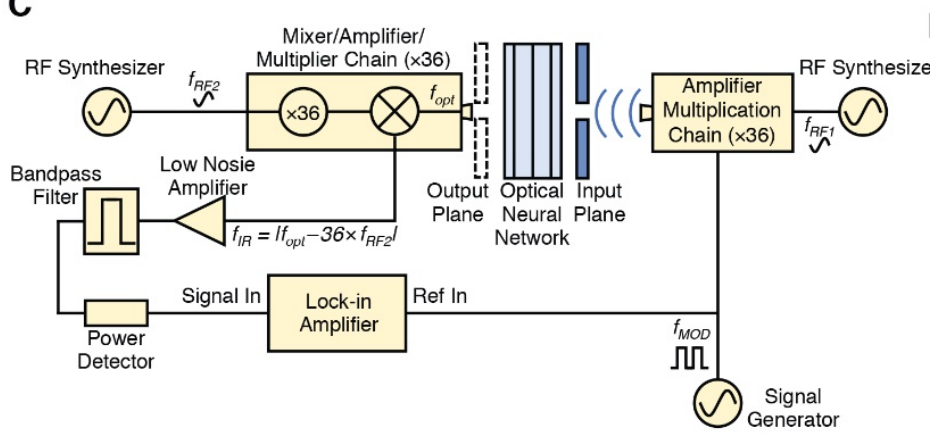

D

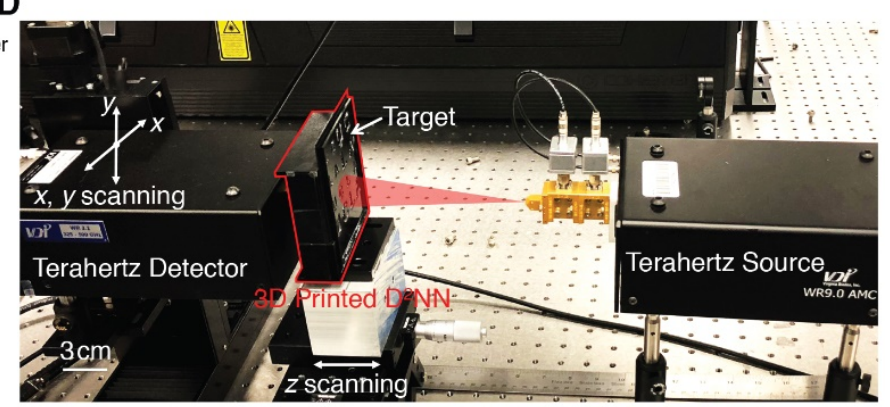

Fig. 2. Experimental testing of 3D-printed $D^{2} N N s$. ( $A$ and $B$ ) After the training phase, the final designs of five different layers $\left(L_{1}, L_{2}, \ldots, L_{5}\right)$ of the handwritten digit classifier, fashion product classifier, and the imaging lens $D^{2} N N s$ are shown. To the right of the network layers, a picture of the corresponding 3D-printed $D^{2} N N$ is shown. A schematic (C) and a picture (D) of the experimental THz setup are shown. An amplifier/multiplier chain was used to generate continuous wave radiation at $0.4 \mathrm{THz}$ and a mixer/amplifier/multiplier chain was used for the detection at the output plane of the network. 
A
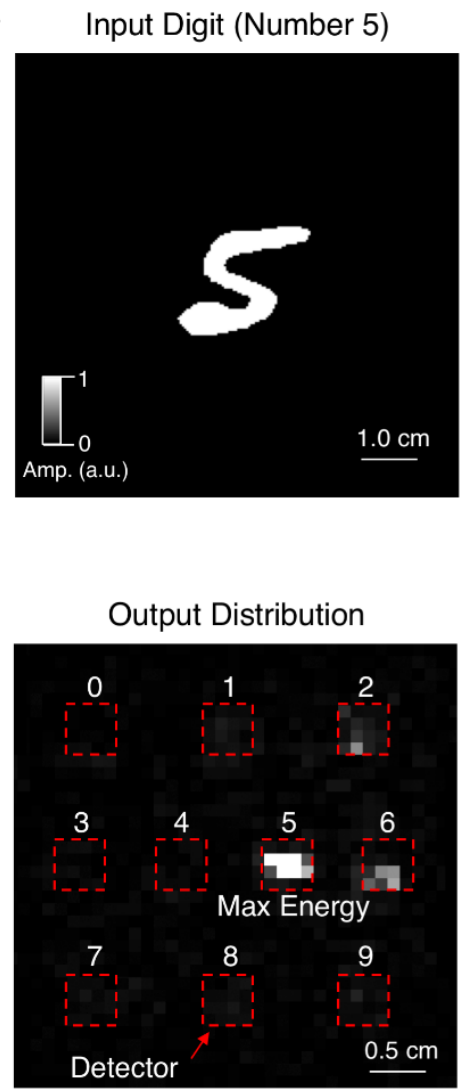

B

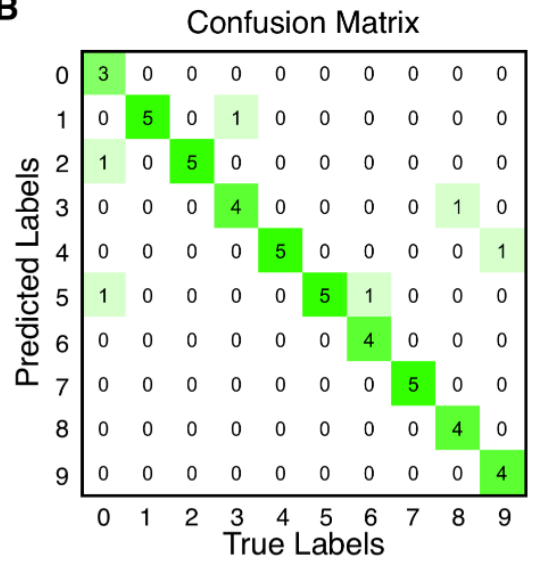

Energy Distribution (Percentage)

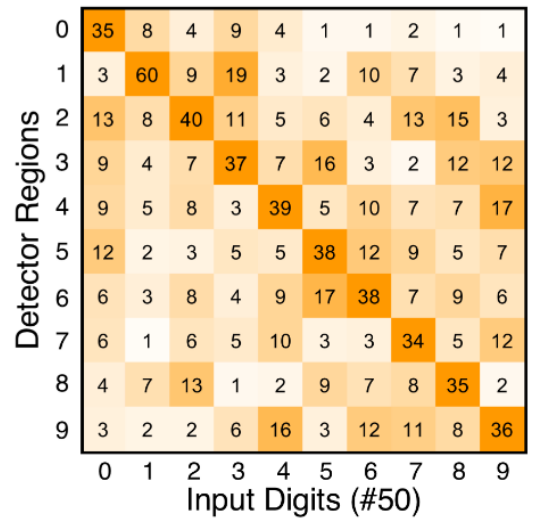

C

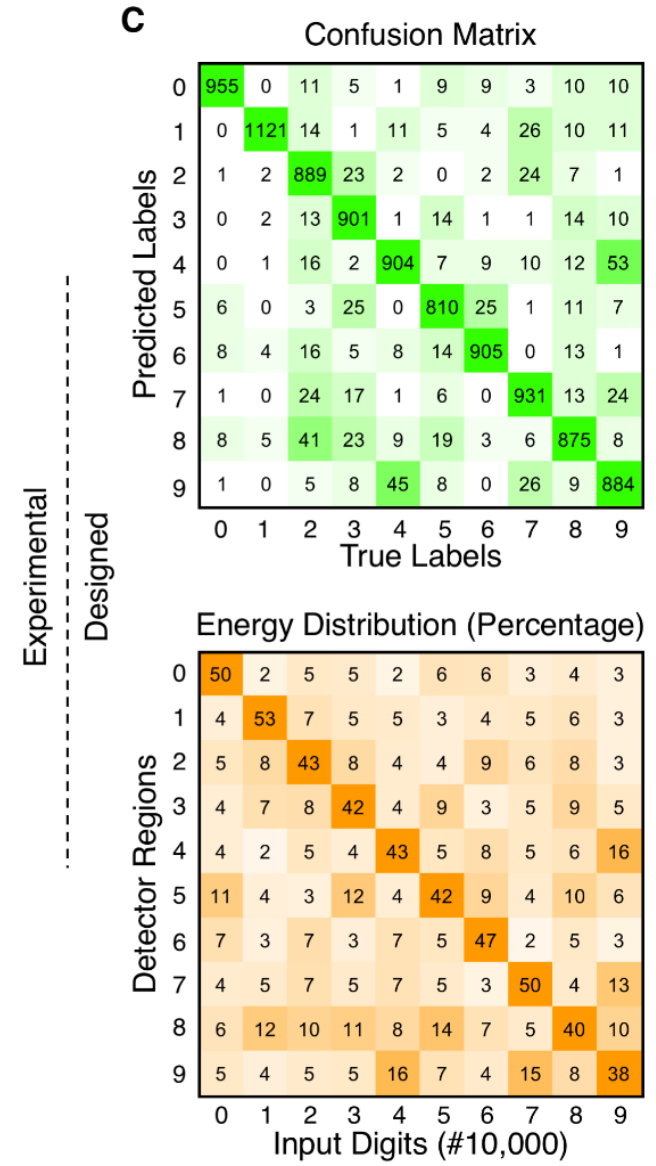

Fig. 3. Handwritten digit classifier $D^{2} N N$. (A) A 3D-printed $D^{2} N N$ successfully classifies handwritten input digits ( 0 , $1, \ldots, 9)$ based on 10 different detector regions at the output plane of the network, each corresponding to one digit. As an example, the output image of the 3D-printed D²NN for a handwritten input of " 5 " is demonstrated, where the red dotted squares represent the trained detector regions for each digit. Other examples of our experimental results are also shown in fig. S9. (B) shows the confusion matrix and the energy distribution percentage for our experimental results, using 50 different handwritten digits that were 3D-printed (i.e., 5 for each digit) selected among the images that numerical testing was successful. (C) is the same as (B), except it summarizes our numerical testing results for 10,000 different handwritten digits ( 1,000 for each digit), achieving a classification accuracy of $91.75 \%$ using a 5layer design. Our classification accuracy increased to $93.39 \%$ by increasing the number of diffractive layers to 7 using a patch of 2 additional diffractive layers to an existing and fixed D²NN (fig. S2). 
A
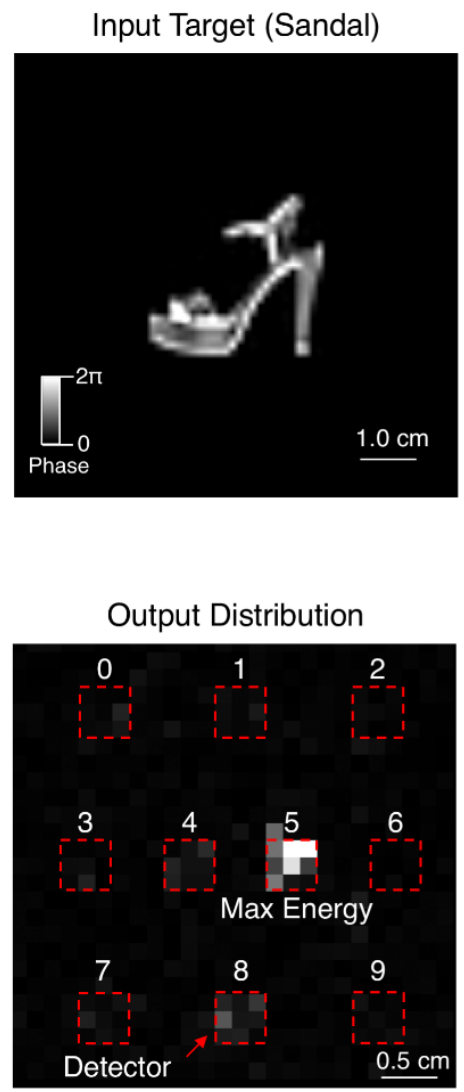

B

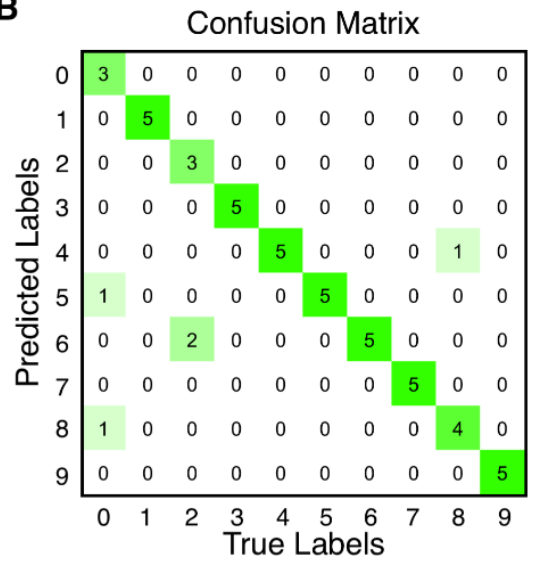

Energy Distribution (Percentage)

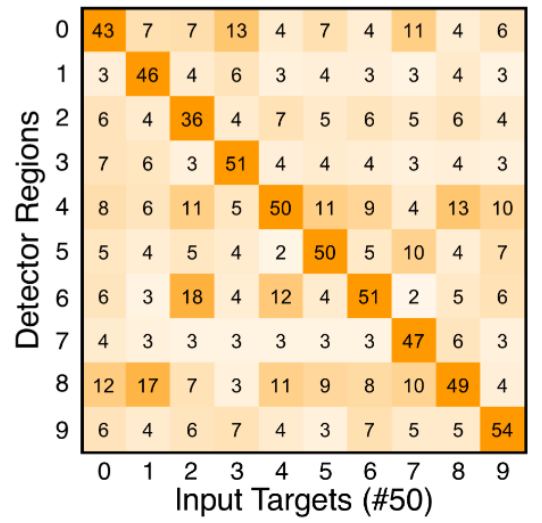

C

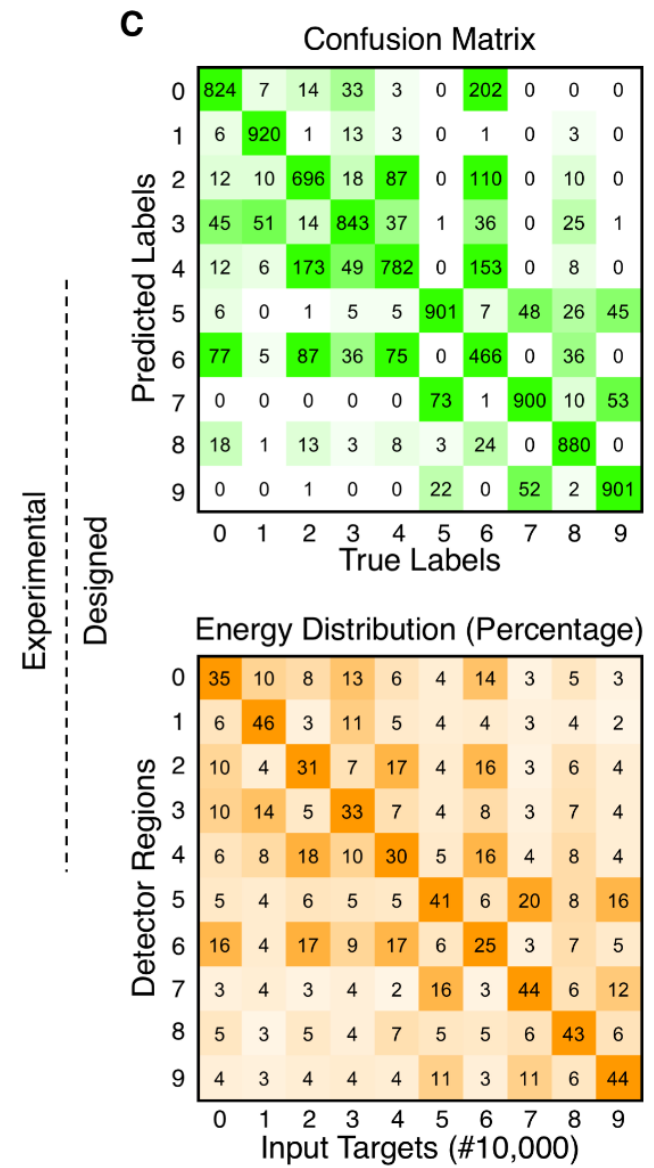

Fig. 4. Fashion product classifier $D^{2} N N$. (A) As an example, the output image of the 3D-printed $D^{2} N N$ for a sandal input (Fashion MNIST class \#5) is demonstrated, where the red dotted squares represent the trained detector regions for each fashion product. Other examples of our experimental results are also shown in fig. S10. (B) shows the confusion matrix and the energy distribution percentage for our experimental results, using 50 different fashion products that were 3D-printed (i.e., 5 per class) selected among the images that numerical testing was successful. (C) is the same as (B), except it summarizes our numerical testing results for 10,000 different fashion products ( 1,000 per class), achieving a classification accuracy of $81.13 \%$ using a 5 -layer design. By increasing the number of diffractive layers to 10 , our classification accuracy increased to $86.60 \%$ (fig. S5). 